\title{
Investigating the Effect of Tumor Microvascular Architecture on Drug Transport and Distribution
}

\author{
Moath Alamer, Xiao Yun Xu \\ Department of Chemical Engineering, Imperial College London \\ South Kensington campus, London, United Kingdom \\ m.alamer15@imperial.ac.uk; yun.xu@imperial.ac.uk
}

\begin{abstract}
In this paper we investigated the transport and distribution of commonly used anti-cancer chemotherapeutics in tumor tissue with explicit representation of the vascular network. Models of the tumor vasculature were generated using a mathematical angiogenesis model. A Green's function-based method was applied to resolve convective and diffusive transport of anti-cancer drugs, doxorubicin and paclitaxel, within the microvascular network and surrounding tissues. The tumor geometry models were validated through morphological and hemodynamic analysis. Our investigation of the transport of different drugs suggest that doxorubicin is limited in its penetration of the tissue to regions near the vessels whilst paclitaxel was able to distribute more effectively in the tissue space. Our simulations of the transport of the drugs in different tumor microvascular networks showed that decreased vascular density resulted in lower drug concentrations. When analyzing the transport in a tumor with a necrotic core, significant spatial gradients in drug concentration were seen between the well vascularized tumor periphery and the avascular tumor center.
\end{abstract}

Keywords: Drug Transport, Tumor Vasculature, Computational Modelling.

\section{Introduction}

Solid tumor tissues exhibit abnormal properties that make it difficult for anti-cancer drugs to transport and reach all cancer cells at adequate concentrations. These properties vary from patient to patient due to the genetic components involved in cancer development which can possibly contribute to differences in response to treatment [1]. One common aberration of tumors is the vasculature which tends to be tortuous and disorganized with erratic changes in diameter and non-uniform distribution. As a result, avascular and necrotic regions with poor perfusion can develop within the tumor. Moreover, individual vessels exhibit abnormalities that render them unstable and leaky leading to elevated interstitial fluid pressure [2]. As most forms of cancer treatments are administered intravenously, these properties can pose barriers to effective drug delivery of anti-cancer drugs. The transport of drugs within the microvessels and interstitial space occurs through a combination of convection and diffusion. The relative importance of each mechanism varies depending on drug properties and fluid velocities which are influenced by physical features of the tumor tissue. In microvessels, convection is usually much faster than diffusion due to the high velocities and length scales whilst in the tissue space, the transport of small solutes would be dominated by diffusion as due to lack of flow [3]. The mechanism by which a drug transports can impact the penetration into the tissue as diffusion is slow and limited to a short distance from the microvessels, whilst convection is much more effective over larger length scales. Hence, there is a need to understand how various drugs with different properties distribute within the tumor tissue under different conditions. Previous models attempted to understand this but have usually treated the vasculature as a uniform source term and neglected the effects of microscopic properties on drug transport. Incorporating the microvasculature of the tumor can help understand the interplay between blood flow, vessel morphology and its effect on drug transport. In this study we attempt to investigate the effect of tumor vasculature on drug transport and distribution at the microscale level by coupling an angiogenesis model with a time-dependent solute transport model. We incorporate microscopic properties through spatial representation of the tumor vasculature with inclusion of individual vessel features such as length and diameter. More so, we study drugs with different properties to understand how they distribute within the tumor tissue and how properties of tumor vasculature influence transport. 


\section{Methodology}

Tumor vascular networks were generated based on the angiogenesis model of Anderson and Chaplain [4]. Different initial conditions were chosen to induce the growth of vascular networks with different architectures and geometries as shown in Figure 1. Blood flow within the tumor vessels was simulated using a Green's Function method developed by Pozrikidis [5]. Morphological and hemodynamic parameters were analyzed for the tumor models and compared to the corresponding data available in the literature [6] as shown in Table 1.
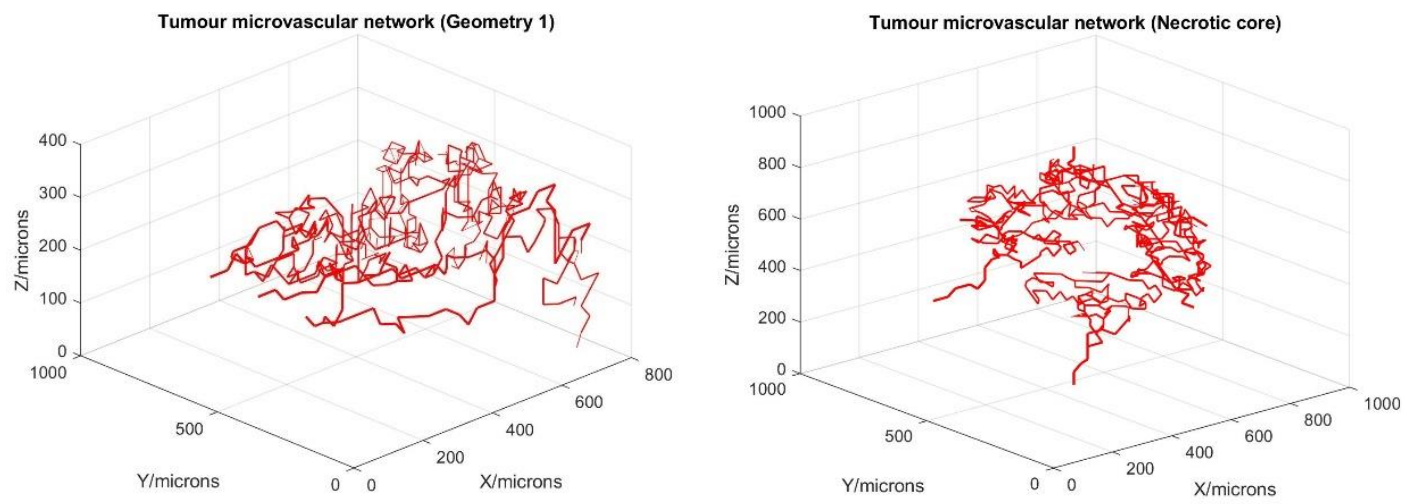

Fig. 1: Different vascular networks generated using mathematical tumor induced angiogenesis model.

Table 1: Comparison of morphological and hemodynamic parameters.

\begin{tabular}{|l|l|c|c|c|}
\hline \multicolumn{1}{|c|}{ Parameter } & \multicolumn{1}{|c|}{ Units } & Geometry 1 & $\begin{array}{c}\text { Necrotic } \\
\text { core }\end{array}$ & $\begin{array}{c}\text { Literature data } \\
\text { (taken from [6]) }\end{array}$ \\
\hline Tumor volume & $\mathrm{mm}^{3}$ & 0.23 & 0.79 & \\
\hline Vascular density & $\%$ & 2.43 & 1.10 & $0.15-1.25$ \\
\hline Maximum extravascular diffusion distance & $\mu \mathrm{m}$ & 65.65 & 106.46 & $30-250$ \\
\hline Mean vessel diameter & $\mu \mathrm{m}$ & 9.22 & 10.89 & $5-225$ \\
\hline Perfusion & $\mathrm{ml} \mathrm{min}^{-1} \mathrm{~g}^{-1}$ & 0.17 & 0.07 & $<2$ \\
\hline
\end{tabular}

\subsection{Drug Transport Model}

To simulate drug transport in these tumor geometries, Secomb's Green's function method for time-dependent solute transport was adopted [7]. The model provides an alternative to direct finite-difference methods which can be computationally intensive when attempting to incorporate the microscopic details of drug transport in tumors. We investigated the effect of solute properties by simulating the transport of therapeutic agents with different transport properties such as doxorubicin and paclitaxel as shown in Table 2. Uptake by cancer cells in the tissue was incorporated and assumed to be linear to reduce complexity. The concentration of drug flowing into the tumor will depend on the amount injected, the infusion time, the drug solubility and how it clears from the body. In this work we ignore these factors and aim to analyze the effects of specific tumor properties on the distribution of anti-cancer drugs. To do this, we assume that doxorubicin and paclitaxel both flow into the tumor at the same concentration of $7 \mu \mathrm{M}$, which was chosen as it is in the range of typical plasma concentrations for both drugs. We discuss the significance of these parameters later in this paper. 
Table 2: Drug parameters in model.

\begin{tabular}{|l|l|c|c|c|}
\hline \multicolumn{1}{|c|}{ Parameter } & \multicolumn{1}{c|}{ Units } & Doxorubicin & Paclitaxel & Reference \\
\hline Diffusivity & $\mathrm{cm}^{2} \mathrm{~s}^{-1}$ & $2 \times 10^{-6}$ & $9 \times 10^{-7}$ & {$[8]$} \\
\hline Reaction/uptake & $\mathrm{s}^{-1}$ & $2.96 \times 10^{-3}$ & $1.19 \times 10^{-4}$ & {$[9,10]$} \\
\hline Molecular weight & $\mathrm{g} \mathrm{mol}^{-1}$ & 543.52 & 853.91 & {$[8]$} \\
\hline
\end{tabular}

\section{Results and Discussion}

Predicted spatial distribution of extravascular drug concentrations in Geometry 1 are presented in Fig. 2 for both drugs. Paclitaxel showed better accumulation where concentration gradients were steeper initially during infusion, however, the gradients became less apparent as time passed and paclitaxel was able to diffuse and distribute more uniformly within the tissue space. Doxorubicin penetration was limited where it was mainly concentrated in regions near the vessels resulting in steeper spatial gradients at all time points. This can be attributed to the difference in uptake rates where doxorubicin has a relatively high net cellular uptake rate within the tissue compared to paclitaxel. Additionally, we analysed intravascular concentrations finding that doxorubicin was heterogeneously distributed and was present at lower concentrations further down the vascular network as shown in Fig. 3. Vessel concentration is influenced by transcapillary exchange which depends on the difference between intravascular and extravascular concentrations. In the case of doxorubicin, larger concentration differences resulting from increased uptake in the tissue space lead to higher flux from vessels, causing limited drug to be available for downstream vessels.
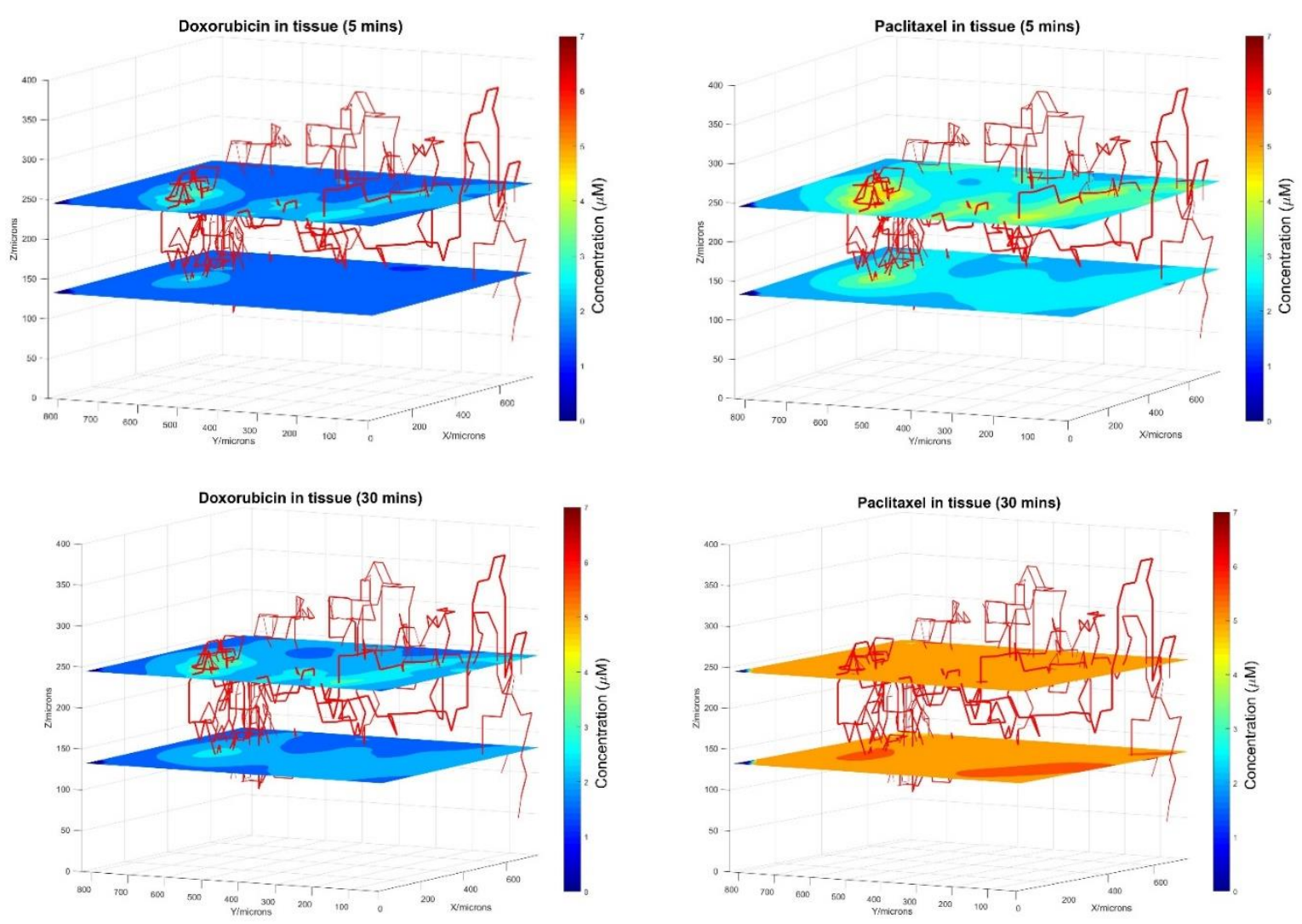

Fig. 2: Extravascular concentration of doxorubicin (left) and paclitaxel (right) in Geometry 1 at different tissue heights after 5 mins (top) and 30 mins (bottom) of continuous infusion. 
To understand the effect of microvascular properties on drug distribution we simulated transport in another vascular network with an avascular necrotic core as shown in Fig. 4. As in the case of geometry 1, paclitaxel was found to accumulate better in the tissue space however, overall, both doxorubicin and paclitaxel accumulation were lower compared to geometry 1. This can be attributed to the higher mean extravascular diffusion distance, lower vascular density and the presence of avascular regions. Analysis of the spatial distribution of the drugs within the tissue space showed that concentrations were higher in the tumor periphery where vasculature was dense whilst the concentrations were lower in poorly vascularized tumor core showing the spatial dependence of drug concentration.
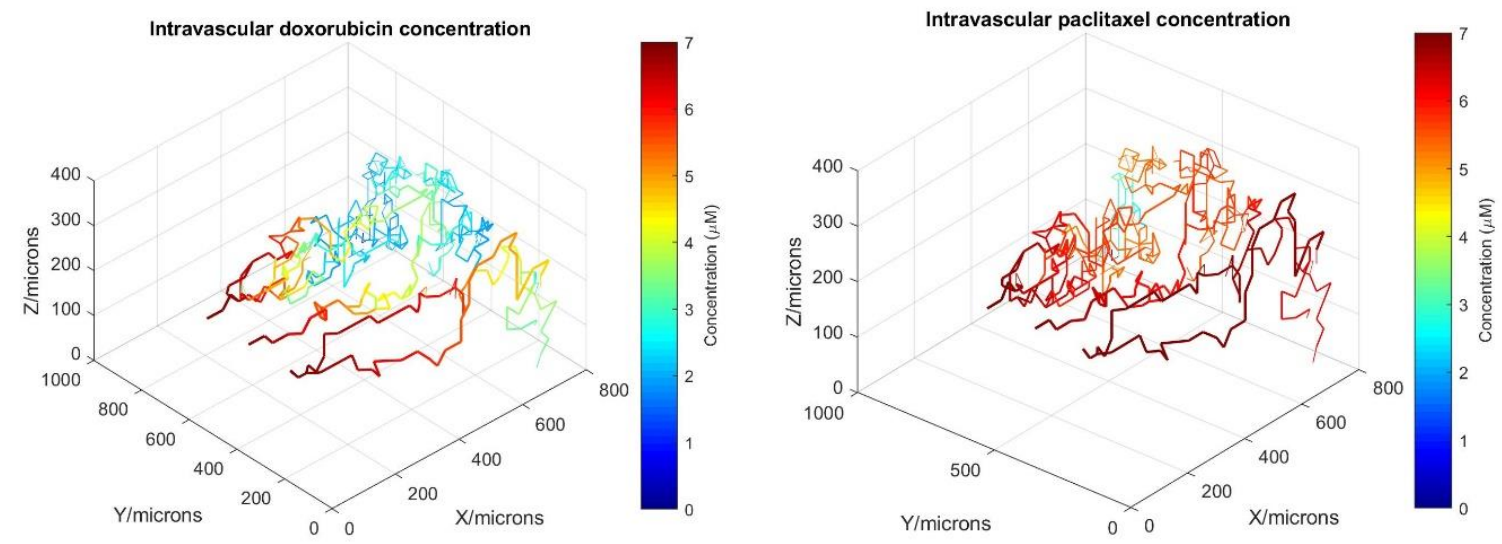

Fig. 3: Intravascular concentration of doxorubicin (left) and paclitaxel (right) after 30 mins of continuous infusion (Geometry 1).
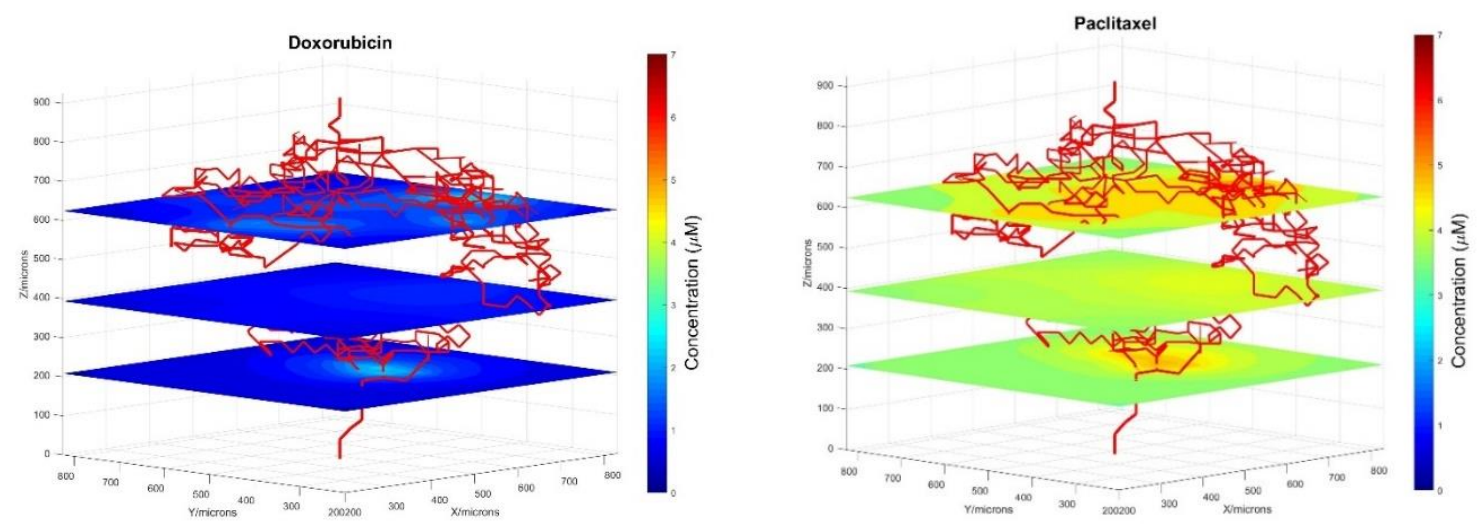

Fig. 4: Extravascular concentration of doxorubicin (left) and paclitaxel (right) at different tissue heights after 30 mins of continuous infusion (Geometry with necrotic core).

\section{Conclusion}

We attempt to show the influence of microscopic properties of the tumor such vessel distribution and network architecture on the transport of drugs within the tissue. Our results suggest that under same conditions, paclitaxel accumulates better within the tumor tissue mainly due to its lower cellular uptake and high penetration depths. Representing heterogeneities in vascular distribution, commonly observed in tumors, affected the transport of both drugs and resulted in steeper spatial gradients of drug concentration. This work highlights the importance of incorporating microscopic properties of the tumor vasculature and providing a spatial representation of drug distribution as this can lead to spatially dependent intracellular uptake and cell killing. This method has also been used to analyse the effect of more properties of the tumor vasculature and blood flow on drug transport which we have not been able to include here. In this work we assumed uptake by cells to be linear to reduce complexity. In reality, the uptake of both drugs is non-linear and is influenced by time and the extracellular concentration of the drug. Uptake rate has been found to increase as extracellular concentration is decreased 
[9][10]. Hence, we envisage that incorporating this non-linearity will further increase heterogeneities in drug distribution within the tumor tissue. Additionally, we expect the concentration of drug flowing into the tumor to play a role in penetration depths.

Further work is being done to incorporate the effect of non-linearity in uptake and how varying administration regimes and infusion rates influence the spatial distribution of the drug, it's peak extravascular concentration and clearance rate. Additionally, a microscopic vascular imaging technique will be coupled with the drug transport model to provide more insight into drug distribution in realistic microvascular networks.

\section{Acknowledgements}

The authors thank Dr Timothy W. Secomb for providing access to the $\mathrm{C}$ program used to implement the drug transport model. This work is supported by funding from UKSACB.

\section{References}

[1] M. R. Junttila and F. J. de Sauvage, "Influence of tumour micro-environment heterogeneity on therapeutic response," Nature, vol. 501, no. 7467, p. 346, 2013.

[2] P. Baluk, H. Hashizume, and D. M. McDonald, "Cellular abnormalities of blood vessels as targets in cancer," Current opinion in genetics \& development, vol. 15, no. 1, pp. 102-111, 2005.

[3] M. W. Dewhirst and T. W. Secomb, "Transport of drugs from blood vessels to tumour tissue," Nature Reviews Cancer, vol. 17, no. 12, p. 738, 2017.

[4] A. R. Anderson and M. Chaplain, "Continuous and discrete mathematical models of tumor-induced angiogenesis," Bulletin of mathematical biology, vol. 60, no. 5, pp. 857-899, 1998.

[5] C. Pozrikidis and D. Farrow, "A model of fluid flow in solid tumors," Annals of biomedical engineering, vol. 31, no. 2, pp. 181-194, 2003.

[6] S. K. Stamatelos, E. Kim, A. P. Pathak, and A. S. Popel, "A bioimage informatics based reconstruction of breast tumor microvasculature with computational blood flow predictions," Microvascular research, vol. 91, pp. 8-21, 2014.

[7] T. W. Secomb, "A Green's function method for simulation of time-dependent solute transport and reaction in realistic microvascular geometries," Mathematical medicine and biology: a journal of the IMA, vol. 33, no. 4, pp. 475-494, 2015.

[8] E. A. Swabb, J. Wei, and P. M. Gullino, "Diffusion and convection in normal and neoplastic tissues," Cancer research, vol. 34, no. 10, pp. 2814-2822, 1974.

[9] D. J. Kerr, A. M. Kerr, R. I. Freshney, and S. B. Kaye, "Comparative intracellular uptake of adriamycin and 4'deoxydoxorubicin by nonsmall cell lung tumor cells in culture and its relationship to cell survival," Biochemical pharmacology, vol. 35, no. 16, pp. 2817-2823, 1986.

[10] E. H. Kerns et al., "Cellular uptake profile of paclitaxel using liquid chromatography tandem mass spectrometry," Rapid communications in mass spectrometry, vol. 12, no. 10, pp. 620-624, 1998. 